\title{
New Formulation for Flexible Beams Undergoing Large Overall Plane Motion
}

\author{
W. J. Haering* \\ General Motors Corporation, Warren, Michigan 48090 \\ R. R. Ryan $\dagger$ \\ Mechanical Dynamics, Inc., Ann Arbor, Michigan 48105 \\ and \\ R. A. Scott \\ University of Michigan, Ann Arbor, Michigan 48109
}

\begin{abstract}
A new flexible body dynamic formulation, called the augmented imbedded geometric constraint approach, for beam structures undergoing large overall motion is developed. It is restricted to small elastic deformations of the beam about the large overall motion. The formulation outlined herein pertains to two-dimensional motion and deformation of a single beam when the overall motion is prescribed as a function of time. The formulation can be easily extended to beam assemblies undergoing arbitrary motion in three-dimensional space. Elastic deformation is characterized by the superposition of a number of assumed global shape functions. The motion of the system is governed by a set of differential and algebraic equations. The algebraic constraints arise from enforcement of the boundary conditions. The new approach improves upon two existing approaches by allowing the solution of two disparate classes of elasto-dynamics problems with a single formulation, demonstrated by simulations for several verification problems. The problems are ones in which the lateral deformation of the beam is dominated by either bending or membrane behavior. Because the new formulation is problem independent, it is applicable to beam problems where the dominant stiffness effects are not known beforehand.
\end{abstract}

\section{Introduction}

T HE study of the coupling between overall dynamic motion and local deformation of structures has become important recently. The effects of such coupling are important in the aeronautics industry and can be seen, for example, in helicopter blade response. High-speed motion of robotic arms and rapid ground transportation systems are other areas in which the coupling effects are important.

One approach to studying flexible body dynamics is through the use of finite element methods (see, for example, Simo, 1 Simo and Vu Quoc, ${ }^{2}$ and Christensen and $\mathrm{Lee}^{3}$ ). Another strategy is to use rigid body dynamic approaches which have been modified to include the flexibility effects. Kane et al. ${ }^{4}$ used this strategy to study beams undergoing large overall motion of a prescribed nature. Banerjee and $\mathrm{Kane}^{\mathrm{s}}$ also used the same strategy to develop a formulation and solutions for plates. In related work, Banerjee and Dickens ${ }^{6}$ used finite element methods to offer an alternative approach, involving arbitrary bodies, to overcome the well-known premature linearization problem, described in Ref. 4.

The technique introduced in Ref. 4 was restricted to systems with known overall motion. Ryan ${ }^{7}$ extended that formulation to allow solutions when forces/torques are applied. Subsequently, Yoo ${ }^{8}$ has shown that the approach in Refs. 4 and 7 , which he refers to as the Imbedded Geometric Constraint (IGC) approach, fails to produce the correct result for prob-

Presented as Paper 92-2261 at the AIAA/ASME/ASCE/AHS/ASC 33rd Structures, Structural Dynamics, and Materials Conference, Dallas, TX, April 13-15, 1992; received June 5, 1992; revision received March 30, 1993; accepted for publication March 30, 1993. Copyright (C) 1992 by the American Institute of Aeronautics and Astronautics, Inc. All rights reserved.

*Senior Research Engineer, Engineering Mechanics Department, General Motors Research and Development Center, Res. Mech. Bldg., 30500 Mound Road. Member AIAA.

$\dagger$ Executive Vice President and Chief Operating Officer, 2301 Commonwealth Blvd. Member AIAA.

$\ddagger$ Professor, Department of Mechanical Engineering and Applied Mechanics, 118 Auto Laboratory. lems where the lateral deformation of the beam is dominated by membrane stiffness. Yoo demonstrated that his formalism, which he refers to as the Nonlinear Strain Displacement (NSD) approach, handles such problems quite successfully. However the NSD method does not reliably solve problems in which lateral deformations are dominated by bending stiffness, which are handled very well by the IGC approach.

A new approach called the Augmented Imbedded Geometric Constraint (AIGC) approach, is presented herein. It allows the solution of problems where the lateral deflection of the beam is dominated by either bending or membrane stiffness. This formulation is a modification of the IGC approach. It is problem independent and, therefore, is applicable to structural dynamics problems where the dominant effects are not known beforehand.

Only small local deformations of the beam are considered. An Euler-Bernoulli model of the beam transverse flexibility, assuming linear elastic, isotropic behavior, is used. A set of ordinary differential equations (ODEs) describing the flexible body dynamic behavior of the beam is developed using the method of Kane and Levinson. ${ }^{9}$ That portion of the development of the AIGC approach is identical to that for the IGC approach. Differential algebraic equations (DAEs) of motion for the AIGC approach are generated by developing a set of algebraic constraints enforcing the physical boundary conditions for the beam. The spatial representation of the deformation is achieved through the use of global shape functions that are based on the substructuring techniques of Craig and Bampton. ${ }^{10}$

\section{Development of System Differential Equations}

The model for a two-dimensional beam undergoing large overall motion and small local deformation is shown in Fig. 1. This model consists of a rigid body $A$ and a flexible beam $B$ of length $L$. A dextral set of mutually perpendicular unit vectors, $a_{1}, a_{2}$, and $a_{3}$, are fixed in $A$ and directed as shown in Fig. 1. The centroidal axis of the beam is assumed to be coincident with the elastic axis, and is parallel to the $a_{1}$ direction when the beam is undeformed. Point $P_{0}$ located a distance $x$ along the 


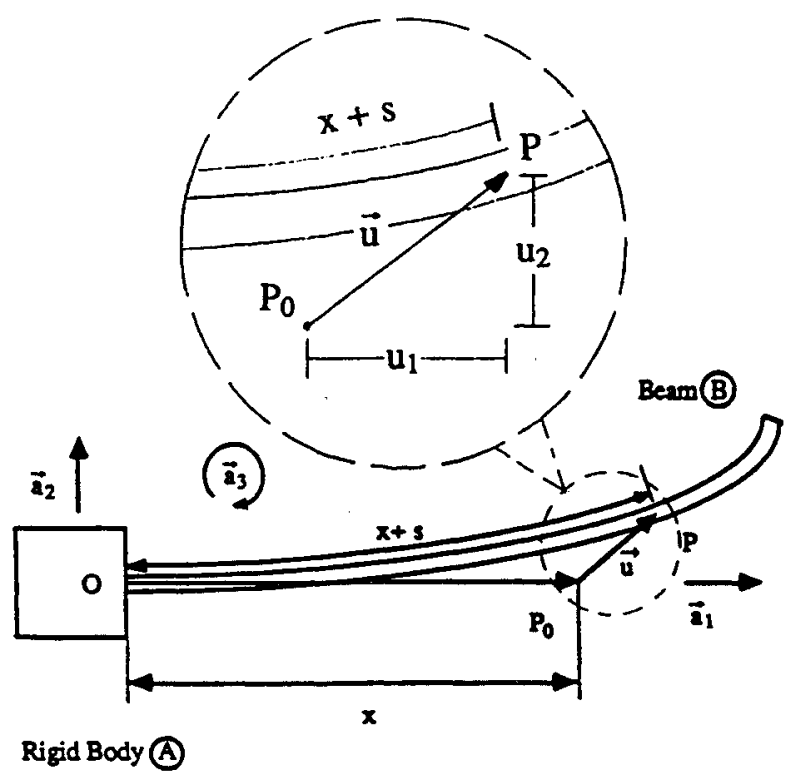

Fig. 1 Beam description and deformation measures.

undeformed centroidal axis represents a generic point on the beam. After deformation, that point lies at a new position which is labeled point $P$. The position vector from point $O$ to point $P$ is given by

$$
p^{\overline{O P}}=\left(x+u_{1}\right) a_{1}+u_{2} a_{2}
$$

where $u_{1}$ and $u_{2}$ are the $a_{1}$ and $a_{2}$ measures of the beam deformation.

An additional variable of interest is $s$, the stretch of the centroidal axis of the beam. After deformation, point $P$ is located at a distance $x+s$ measured along the deformed centroidal axis.

The equations of motion are derived using Kane's dynamical equations:

$$
F_{r}+F_{r}^{*}=0, \quad r=1,2, \ldots, p
$$

where $p$ is the number of system degrees of freedom, and $F_{r}$ and $F_{r}^{*}$ are the $r$ th generalized active force and generalized inertia force, respectively.

The generalized inertia force $F_{r}^{*}$ is associated with the mass distributed along the length of the beam and is given by

$$
F_{r}^{*}=-\int_{0}^{L} \rho^{N} \boldsymbol{v}_{r}^{P} \cdot N^{P}{ }^{P} \mathrm{~d} x, \quad r=1,2, \ldots, p
$$

where $\rho$ represents the beam mass per unit length and ${ }^{N} a^{P}$ represents the acceleration of point $P$ in the Newtonian reference frame $N$. The ${ }^{N} \boldsymbol{v}_{r}^{P}$ is the $r$ th partial velocity of ${ }^{N} v^{P}$, the velocity of point $P$ in the Newtonian reference frame $N$, and is a fundamental element in the formulation of Kane's dynamical equations. The partial velocity is defined as

$$
N_{\boldsymbol{v}_{r}^{P}} \equiv \frac{\partial^{N} \boldsymbol{v}^{P}}{\partial u_{r}^{*}}, \quad r=1,2, \ldots, p
$$

where $u_{r}^{*}$ are the generalized speeds. In this development they are chosen as

$$
u_{r}^{*}=\dot{q}_{r}, \quad r=1,2, \ldots, p
$$

where $q_{r}$ is the $r$ th generalized coordinate (specified later), and a dot denotes a time derivative.
The generalized active force, $F_{r}$, derived from a potential function describing the strain energy $V$ is

$$
F_{r}=-\frac{\partial V}{\partial q_{r}}, \quad r=1,2, \ldots, p
$$

Detailed expressions for the generalized inertia and generalized active forces will now be developed.

\section{Generalized Inertia Force}

As seen in Eq. (3), the acceleration and velocity of point $P$ in a Newtonian reference frame are needed to form the generalized inertia force. To facilitate their formulation, the planar motion of the rigid base, relative to the Newtonian reference frame, can be described by its angular velocity, ${ }^{N} \omega^{A}$, and the translational velocity of some point $O,{ }^{N} v^{O}$, fixed on the base.

$$
\begin{gathered}
N_{\omega^{A}=\omega_{3} a_{3}} \\
N_{\boldsymbol{v}} O=v_{1} a_{1}+v_{2} a_{2}
\end{gathered}
$$

where $\omega_{3}$ is the $a_{3}$ measure of the angular velocity of body $A$, and $v_{1}$ and $v_{2}$ are the $\boldsymbol{a}_{1}$ and $\boldsymbol{a}_{2}$ measures of the translational velocity of point $O$.

The velocity of point $P$ in the Newtonian reference frame ${ }^{N} v^{P}$ is

$$
{ }^{N} \boldsymbol{v}^{P}=\left[v_{1}+\dot{u}_{1}-\omega_{3} u_{2}\right] \boldsymbol{a}_{1}+\left[v_{2}+\dot{u}_{2}+\omega_{3}\left(x+u_{1}\right)\right] \boldsymbol{a}_{2}
$$

The Newtonian acceleration of point $P,{ }^{N} a^{P}$, is

$$
\begin{gathered}
N^{N} \boldsymbol{a}^{P}=\left[\dot{v}_{1}+\ddot{u}_{1}-\dot{\omega}_{3} u_{2}-2 \omega_{3} \dot{u}_{2}-\omega_{3} v_{2}-\omega_{3}^{2}\left(x+u_{1}\right)\right] \boldsymbol{a}_{1} \\
\quad+\left[\dot{v}_{2}+\ddot{u}_{2}+\dot{\omega}_{3}\left(x+u_{1}\right)+2 \omega_{3} \dot{u}_{1}+\omega_{3} v_{1}-\omega_{3}^{2} u_{2}\right] \boldsymbol{a}_{2}
\end{gathered}
$$

In the current (as well as the IGC) approach, the variables $s$ and $u_{2}$ are used to describe the deformation. They are represented as follows:

$$
\begin{aligned}
& s=\sum_{j=1}^{\nu} \phi_{1 j}(x) q_{j}(t) \\
& u_{2}=\sum_{j=1}^{\nu} \phi_{2 j}(x) q_{j}(t)
\end{aligned}
$$

where $\phi_{1 j}$ and $\phi_{2 j}$ are shape functions, $q_{j}$ are the generalized coordinates, and $\nu$ is the total number of functions used to describe the beam deformation. The specific choice of the shape functions, $\phi_{1 j}$ and $\phi_{2 j}$ will be presented later.

Since $s$ and not $u_{1}$ is chosen as a deformation measure, the following geometric relationship, initially given in Ref. 8 (development can be found in Haering ${ }^{11}$ ), between the variables $s$, $u_{1}$, and $u_{2}$ will be used to develop expressions for $u_{1}, \dot{u}_{1}$, and $\ddot{u}_{1}$ which appear in Eqs. (9) and (10)

$$
x+s=\int_{0}^{x}\left[\left(1+\frac{\partial u_{1}}{\partial \sigma}\right)^{2}+\left(\frac{\partial u_{2}}{\partial \sigma}\right)^{2}\right]^{1 / 2} \mathrm{~d} \sigma
$$

where $\sigma$ is a dummy variable of integration. After performing a binomial expansion on the right-hand side of Eq. (13) and neglecting terms higher than degree two, the following expression can be obtained

$$
u_{1}=s-\frac{1}{2} \int_{0}^{x}\left(\frac{\partial u_{2}}{\partial \sigma}\right)^{2} \mathrm{~d} \sigma
$$

Substituting the relationships for $s$ and $u_{2}$ from Eqs. (11) and (12) into Eq. (14) yields

$$
u_{1}=\sum_{j=1}^{\nu} \phi_{1 j} q_{j}-\frac{1}{2} \int_{0}^{x} \sum_{j=1}^{\nu} \sum_{k=1}^{\nu} \phi_{2 j}^{\prime} \phi_{2 k}^{\prime} q_{j} q_{k} \mathrm{~d} \sigma
$$


where a prime denotes a partial derivative with respect to $x$. From Eqs. (12) and (15), it follows that

$$
\begin{gathered}
\dot{u}_{1}=\sum_{j=1}^{\nu} \phi_{1 j} \dot{q}_{j}-\int_{0}^{x} \sum_{j=1}^{\nu} \sum_{k=1}^{\nu} \phi_{2 j}^{\prime} \phi_{2 k}^{\prime} q_{j} \dot{q}_{k} \mathrm{~d} \sigma \\
\ddot{u}_{1}=\sum_{j=1}^{\nu} \phi_{1 j} \ddot{q}_{j}-\int_{0}^{x} \sum_{j=1}^{\nu} \sum_{k=1}^{\nu} \phi_{2 j}^{\prime} \phi_{2 k}^{\prime} \dot{q}_{j} \dot{q}_{k} \mathrm{~d} \sigma \\
-\int_{0}^{x} \sum_{j=1}^{\nu} \sum_{k=1}^{\nu} \phi_{2 j}^{\prime} \phi_{2 k}^{\prime} q_{j} \ddot{q}_{k} \mathrm{~d} \sigma \\
\dot{u}_{2}=\sum_{j=1}^{\nu} \phi_{2 j} \dot{q}_{j} \\
\ddot{u}_{2}=\sum_{j=1}^{\nu} \phi_{2 j} \ddot{q}_{j}
\end{gathered}
$$

Recalling Eq. (4), the partial velocities of point $P$ in frame $N$, $N^{*}{ }_{i}^{P}$, are given by

$$
\begin{array}{r}
N_{i}^{P}=\left[\phi_{1 i}-\sum_{j=1}^{\nu}\left(\int_{0}^{x} \phi_{2 i}^{\prime} \phi_{2 j}^{\prime} \mathrm{d} \sigma\right) q_{j}\right] \boldsymbol{a}_{1}+\phi_{2 i} \boldsymbol{a}_{2} \\
i=1, \ldots, \nu
\end{array}
$$

The generalized inertia force is developed from Eqs. (3), (10), and (20).

$$
\begin{aligned}
F_{i}^{*} & =-\int_{0}^{L} \rho\left\{\phi_{1 i}\left[\dot{v}_{1}+\ddot{u}_{1}-\dot{\omega}_{3} u_{2}-2 \omega_{3} \dot{u}_{2}-\omega_{3} v_{2}-\omega_{3}^{2}\left(x+u_{1}\right)\right]\right. \\
& -\left[\sum_{j=1}^{\nu}\left(\int_{0}^{x} \phi_{2 i}^{\prime} \phi_{2 j}^{\prime} \mathrm{d} \sigma\right) q_{j}\right]\left[\dot{v}_{1}+\ddot{u}_{1}-\dot{\omega}_{3} u_{2}-2 \omega_{3} \dot{u}_{2}\right. \\
& \left.-\omega_{3} v_{2}-\omega_{3}^{2}\left(x+u_{1}\right)\right]+\phi_{2 i}\left[\dot{v}_{2}+\ddot{u}_{2}+\dot{\omega}_{3}\left(x+u_{1}\right)\right. \\
& \left.\left.+2 \omega_{3} \dot{u}_{1}+\omega_{3} v_{1}-\omega_{3}^{2} u_{2}\right]\right\} \mathrm{d} x, \quad i=1,2, \ldots, v
\end{aligned}
$$

Substitution of the expressions in Eqs. (12) and (15-19) into Eq. (21) results in nonlinear terms in $q_{j}$ and $\dot{q}_{j}$. Because only small elastic deformations are of interest, the resulting expression for the generalized inertia force is linearized in $q_{j}$ and $\dot{q}_{j}$. (The validity of this linearization was investigated in Ref. 11 for one of the problems addressed in this paper. No observable difference was found when the solution to differential equations, developed by retaining up to third degree nonlinearties in $q_{j}$ and $\dot{q}_{j}$, was compared with the one from the linearized equations.) Also, integration by parts (see Ref. 11 for details) is applied to eliminate the indefinite integrals arising from the expressions for $u_{1}, \dot{u}_{1}$, and $\ddot{u}_{1}$.

The final simplified form of the generalized inertia force is

$$
\begin{gathered}
-F_{i}^{*}=\left(\dot{v}_{1}-\omega_{3} v_{2}\right) \bar{W}_{1 i}+\left(\dot{v}_{2}+\omega_{3} v_{1}\right) \bar{W}_{2 i}-\omega_{3}^{2} \bar{X}_{1 i}+\dot{\omega}_{3} \bar{X}_{2 i} \\
+\sum_{j=1}^{\nu}\left[W_{11 i j}+W_{22 i j}\right] \ddot{q}_{j}+2 \omega_{3} \sum_{j=1}^{\nu}\left[W_{21 i j}-W_{12 i j}\right] \dot{q}_{j} \\
-\omega_{3}^{2} \sum_{j=1}^{\nu}\left[W_{11 i j}+W_{22 i j}\right] q_{j}+\dot{\omega}_{3} \sum_{j=1}^{\nu}\left[W_{21 i j}-W_{12 i j}\right] q_{j} \\
-\left(\dot{v}_{1}-\omega_{3} v_{2}\right) \sum_{j=1}^{\nu} I P 1_{i j} q_{j}+\omega_{3}^{2} \sum_{j=1}^{\nu} I P 2_{i j} q_{j} \\
i=1,2, \ldots, \nu
\end{gathered}
$$

where

$$
\begin{gathered}
\bar{W}_{k i} \equiv \int_{0}^{L} \rho \phi_{k i} \mathrm{~d} x \\
W_{k m i j} \equiv \int_{0}^{L} \rho \phi_{k i} \phi_{\mathrm{mj}} \mathrm{d} x
\end{gathered}
$$

$$
\bar{X}_{k i} \equiv \int_{0}^{L} \rho x \phi_{k i} \mathrm{~d} x
$$

and terms resulting from the aforementioned integration by parts are

$$
\begin{gathered}
I P 1_{i j}=\rho \int_{0}^{L}(L-x) \phi_{2 i}^{\prime} \phi_{2 j}^{\prime} \mathrm{d} x \\
I P 2_{i j}=\frac{1}{2} \rho \int_{0}^{L}\left(L^{2}-x^{2}\right) \phi_{2 i}^{\prime} \phi_{2 j}^{\prime} \mathrm{d} x
\end{gathered}
$$

\section{Generalized Active Force and System Differential Equations}

Only axial and bending contributions to the strain energy function introduced in Eq. (6) will be considered. Using EulerBernoulli beam theory and assuming uniform axial stress the total strain energy $V$ can be expressed as

$$
V=\frac{1}{2} \int_{0}^{L} E A_{b}\left(\frac{\partial s}{\partial x}\right)^{2} \mathrm{~d} x+\frac{1}{2} \int_{0}^{L} E I_{3}\left(\frac{\partial^{2} u_{2}}{\partial x^{2}}\right)^{2} \mathrm{~d} x
$$

where $E$ is Young's modulus, $I_{3}$ is the area moment of inertia of the beam about the $a_{3}$ axis, and $A_{b}$ is the cross-sectional area of the beam.

After appropriate substitution, partial differentiation, and rearranging, the final form of the generalized active force is

$$
\begin{gathered}
-F_{i}=\sum_{j=1}^{\nu}\left[\int_{0}^{L} E A_{b} \phi_{1 i}^{\prime} \phi_{1 j}^{\prime} \mathrm{d} x\right] q_{j}+\sum_{j=1}^{\nu}\left[\int_{0}^{L} E I_{3} \phi_{2 i}^{\prime \prime} \phi_{2 j}^{\prime \prime} \mathrm{d} x\right] q_{j} \\
i=1,2, \ldots, \nu
\end{gathered}
$$

Using Kane's dynamical equations [Eq. (2)], the resulting system differential equations are

$$
\begin{gathered}
\sum_{j=1}^{\nu}\left[W_{11 i j}+W_{22 i j}\right] \ddot{q}_{j}+2 \omega_{3} \sum_{j=1}^{\nu}\left[W_{21 i j}-W_{12 i j}\right] \dot{q}_{j} \\
-\omega_{3}^{2} \sum_{j=1}^{\nu}\left[W_{11 i j}+W_{22 i j}\right] q_{j}+\dot{\omega}_{3} \sum_{j=1}^{\nu}\left[W_{21 i j}-W_{12 i j}\right] q_{j} \\
-\left(\dot{v}_{1}-\omega_{3} v_{2}\right) \sum_{j=1}^{\nu} I P 1_{i j} q_{j}+\omega_{3}^{2} \sum_{j=1}^{\nu} I P 2_{i j} q_{j}+\sum_{j=1}^{\nu} H_{i j} q_{j} \\
=-\left(\dot{v}_{1}-\omega_{3} v_{2}\right) \bar{W}_{1 i}-\left(\dot{v}_{2}+\omega_{3} v_{1}\right) \bar{W}_{2 i}+\omega_{3}^{2} \bar{X}_{1 i}-\dot{\omega}_{3} \bar{X}_{2 i} \\
i=1,2, \ldots, \nu
\end{gathered}
$$

where

$$
H_{i j}=\int_{0}^{L} E A_{b} \phi_{1 i}^{\prime} \phi_{1 j}^{\prime} \mathrm{d} x+\int_{0}^{L} E I_{3} \phi_{2 i}^{\prime \prime} \phi_{2 j}^{\prime \prime} \mathrm{d} x
$$

In the AIGC approach a set of algebraic constraints is added to the system differential equations to form the equations of motion. The algebraic constraints are introduced to overcome the failure of the IGC approach to insure satisfaction of the boundary conditions for membrane-dominated problems. Additional insight into the utility of the algebraic constraints can be gained by investigating the failure of the NSD approach to insure satisfaction of the boundary conditions for bendingdominated problems.

\section{Enforcement of Boundary Conditions}

For the bending-stiffness-dominated beam problem depicted in Fig. 2, the shape functions are obtained from solving axial and bending vibration problems with the respective boundary conditions

$$
\begin{gathered}
\left.\phi_{1 j}\right|_{x=0}=0 \\
\left.\frac{\partial \phi_{1 j}}{\partial x}\right|_{x=L}=0
\end{gathered}
$$




$$
\begin{gathered}
\left.\phi_{2 j}\right|_{x=0}=0 \\
\left.\frac{\partial^{2} \phi_{2 j}}{\partial x^{2}}\right|_{x=L}=0 \\
\left.\frac{\partial \phi_{2 j}}{\partial x}\right|_{x=0}=0 \\
\left.\frac{\partial^{3} \phi_{2 j}}{\partial x^{3}}\right|_{x=L}=0
\end{gathered}
$$

Using these choices for the shape functions in Eqs. (11) and (12) (IGC approach), it is seen that the boundary conditions set forth in Fig. 2 are fully satisfied. On the other hand, in the initial NSD approach, the deformation measures were taken to be

$$
\begin{aligned}
& u_{1}=\sum_{j=1}^{\nu} \phi_{1 j}(x) q_{j}(t) \\
& u_{2}=\sum_{j=1}^{\nu} \phi_{2 j}(x) q_{j}(t)
\end{aligned}
$$

with $\phi_{1 j}$ and $\phi_{2 j}$ as before. Hence, in this representation, instead of enforcing $\partial s /\left.\partial x\right|_{x=L}=0$, the condition $\partial u_{1} /\left.\partial x\right|_{x=L}=0$ is enforced. Yoo modified his initial NSD approach to improve the poor modal convergence of the approach for bending-stiffness-dominated problems. He did this through the use of socalled interaction modes (between $u_{1}$ and $u_{2}$ ) generated by enforcing an inextensibility condition. While some improvement was found, the final approach still does not satisfy the aforementioned boundary condition and does not yield an accurate solution for some circumstances (as will be shown shortly).

For the membrane-stiffness-dominated beam problem shown in Fig. 3, the shape functions are obtained from vibration analyses in which the following boundary conditions are enforced.

$$
\begin{gathered}
\left.\phi_{1 j}\right|_{x=0}=0 \\
\left.\phi_{1 j}\right|_{x=L}=0 \\
\left.\phi_{2 j}\right|_{x=0}=0 \\
\left.\phi_{2 j}\right|_{x=L}=0 \\
\left.\frac{\partial^{2} \phi_{2 j}}{\partial x^{2}}\right|_{x=0}=0
\end{gathered}
$$

$$
\left.\frac{\partial^{2} \phi_{2 j}}{\partial x^{2}}\right|_{x=L}=0
$$

Thus the NSD approach, using these shape functions in Eqs. (38) and (39), fully satisfies the boundary conditions shown in Fig. 3. Note that in the IGC approach the condition $\left.s\right|_{x=L}=0$ is enforced instead of $\left.u_{1}\right|_{x=L}=0$.

By using the shape function summation approach, only boundary conditions explicit in the chosen deformation measures can be enforced. Thus only boundary conditions expressed in $s$ and $u_{2}$ can be enforced for the IGC approach and only boundary conditions expressed in $u_{1}$ and $u_{2}$ can be enforced for the NSD approach. This prevents either approach from accurately addressing both the bending and membrane problems, without some modification.

Boundary conditions which are not explicit in the chosen deformation measures can be enforced by adding constraints derived from evaluation of Eq. (15), which represents the geometric relationship between $u_{1}, s$, and $u_{2}$. Recall that in the IGC approach, the condition that $u_{1}$ be zero at the right end of the beam (membrane-dominated problem) could not be satisfied. Using Eq. (15) this boundary condition becomes the following constraint between the generalized coordinates:

$$
0=\sum_{j=1}^{\nu} \phi_{1 j}(L) q_{j}-\frac{1}{2} \sum_{j=1}^{\nu} \sum_{k=1}^{\nu}\left[\int_{0}^{L} \phi_{2 j}^{\prime} \phi_{2 k}^{\prime} \mathrm{d} x\right] q_{j} q_{k}
$$

This is an example of the general approach for enforcing boundary conditions. However, care must be taken in selecting the shape functions so that undesirable conditions do not arise. For example, using the shape functions just described in the IGC approach, the constraint [Eq. (46)] could, possibly, be satisfied. However, zero stretch at the right end of the beam would also result, which is not physically correct. Clearly what is needed is an approach that allows arbitrary boundary conditions to be prescribed. This is achieved by using an approach similar to one set forth by Craig and Bampton in work on dynamic system substructuring. ${ }^{10}$ This will now be described.

\section{Shape Functions, Constraints, and Complete Equations of Motion}

Craig and Bampton use dynamic modes in combination with static displacement modes in a substructuring approach for vibration problems. The dynamic modes, referred to as fixedinterface modes, are developed from an eigenanalysis of the lateral vibrations of a beam with boundary conditions of zero slope and displacement at the ends of the beam. The static

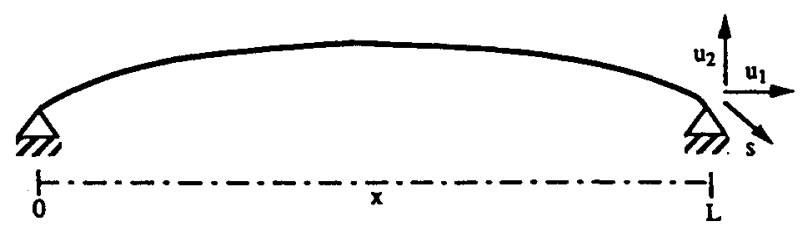

Boundary Conditions

$$
\begin{array}{ll}
\left.u_{1}\right|_{x=0}=0, \text { or } s b_{x=0}=0 & u_{1} k_{x=L}=0 \\
\left.u_{2}\right|_{x=0}=0 & \left.u_{2}\right|_{x=L}=0 \\
\left.\frac{\partial^{2} u_{2}}{\partial x^{2}}\right|_{x=0}=0 & \left.\frac{\partial^{2} u_{2}}{\partial x^{2}}\right|_{x=L}=0
\end{array}
$$

Fig. 2 Boundary conditions for a cantilever beam.

Fig. 3 Boundary conditions for a pinned-pinned beam. 
modes, referred to as constraint modes, are obtained by applying unit displacements or rotations in the directions held fixed in developing the dynamic modes. While enforcing each unit displacement or rotation, the others are held fixed. For example, one such shape function satisfies the conditions $\phi(0)=1$ and $\phi^{\prime}(0)=\phi(L)=\phi^{\prime}(L)=0$. The combined set of shape functions, static and dynamic, is capable of representing any arbitrary boundary condition. The approaches for developing the modes to describe the $s$ and $u_{2}$ deformations are slightly different and are outlined below.

The dynamic shape functions for the $u_{2}$ deformation are the eigenfunctions obtained from a linear lateral vibration analysis of a beam in a nonrotating frame with zero displacement and slope at both ends. The static shape functions are polynomials developed to satisfy the enforced unit displacement or slope conditions. Third degree polynomials are used because four boundary conditions need to be satisfied. The four static shape functions for lateral deformation are given by

$$
\begin{gathered}
\phi_{\text {static 1 }}=2.0\left[0.5-1.5(x / L)^{2}+(x / L)^{3}\right] \\
\phi_{\text {static 3 }}=2.0\left[1.5(x / L)^{2}-(x / L)^{3}\right] \\
\phi_{\text {static 2 }}=x / L-2.0(x / L)^{2}+(x / L)^{3} \\
\phi_{\text {static 4 }}=-(x / L)^{2}+(x / L)^{3}
\end{gathered}
$$

The development of the modal functions to describe the $s$ deformation is as follows. The dynamic shape functions are derived from an eigenanalysis of the axial vibrations of a rod with no displacement at both ends. Initially, two static modes were developed from polynomials which satisfy unit displacement at one end and zero displacement at the other end. However, numerical simulations indicated much better modal convergence when the four lateral static modal functions are used instead. Thus, the same static shape functions are used for the $s$ and $u_{2}$ direction deformation representations.

The constraint equations for the bending and membrane problems are now set forth.

1) Bending constraints:

$$
\begin{aligned}
& \sum_{j=1}^{\nu} \phi_{1 j}(0) q_{j}=0 \\
& \sum_{j=1}^{\nu} \phi_{2 j}(0) q_{j}=0 \\
& \sum_{j=1}^{\nu} \phi_{2 j}^{\prime}(0) q_{j}=0 \\
& \sum_{j=1}^{\nu} \phi_{1 j}^{\prime}(L) q_{j}=0 \\
& \sum_{j=1}^{\nu} \phi_{2 j}^{\prime \prime \prime}(L) q_{j}=0 \\
& \sum_{j=1}^{\nu} \phi_{2 j}^{\prime \prime}(L) q_{j}=0
\end{aligned}
$$

2) Membrane constraints:

$$
\begin{gathered}
\sum_{j=1}^{\nu} \phi_{1 j}(0) q_{j}=0 \\
\sum_{j=1}^{\nu} \phi_{2 j}(0) q_{j}=0 \\
\sum_{j=1}^{\nu} \phi_{2 j}^{\prime \prime}(0) q_{j}=0 \\
\sum_{j=1}^{\nu} \phi_{1 j}(L) q_{j}-\frac{1}{2} \sum_{j=1}^{\nu} \sum_{k=1}^{\nu}\left[\int_{0}^{L} \phi_{2 j}^{\prime} \phi_{2 k}^{\prime} \mathrm{d} x\right] q_{j} q_{k}=0
\end{gathered}
$$

$$
\begin{aligned}
& \sum_{j=1}^{\nu} \phi_{2 j}(L) q_{j}=0 \\
& \sum_{j=1}^{\nu} \phi_{2 j}^{\prime \prime}(L) q_{j}=0
\end{aligned}
$$

Thus the complete differential algebraic equations of motion for the membrane problem using the AIGC approach are the ODEs in Eq. (30), along with the constraints in Eqs. (51a-51f). For the membrane problem, the ODEs in Eq. (30), in addition to the constraints in Eqs. (52a-52f), describe the system.

Despite the fact that the AIGC approach provides different constraints for the bending and membrane problems [Eqs. (51) and (52), respectively], the approach is problem independent. This is true because the constraints are handled numerically in a generic way, and therefore differences in the specific constraints do not affect the solution procedure. That different boundary conditions must be specified for each given problem is simply part of the problem description and is necessary in any approach.

Here, numerical solutions to the differential algebraic equations of motion are obtained using the approach developed by Baumgarte. ${ }^{12}$ Baumgarte's approach involves two basic steps, namely appending the constraint equations $\left(\Phi_{j}=0, j=1,2\right.$, $\ldots, r$, where $r$ is the number of constraints) to the differential equations via Lagrange multipliers and differentiating the constraints. The process yields equations of the following form, if index notation is dropped (temporarily)

$$
\left[\begin{array}{cc}
M & \Phi_{q}^{T} \\
\Phi_{q} & 0
\end{array}\right]\left\{\begin{array}{l}
\ddot{q} \\
\lambda
\end{array}\right\}=\left\{\begin{array}{c}
F-G \dot{q}-K q \\
R
\end{array}\right\}
$$

The symbols $M, G, K$, and $F$ represent the mass, gyroscopic, stiffness, and force matrices from the original differential equations; $q, \dot{q}$, and $\ddot{q}$ are column matrices of the generalized coordinates and their time derivatives; $\lambda$ is a column matrix of the Lagrange multipliers; and the column matrix $R$ is given by

$$
R=-(\dot{\Phi})_{q} \dot{q}-\Phi_{t t}-\alpha \dot{\Phi}-\beta^{2} \Phi
$$

which fully written out in index notation is

$$
\begin{aligned}
R_{j} & =-\sum_{i=1}^{n} \frac{\partial\left(\sum_{k=1}^{n} \frac{\partial \Phi_{j}}{\partial q_{k}} \dot{q}_{k}+\frac{\partial \Phi_{j}}{\partial t}\right)}{\partial q_{i}} \dot{q}_{i}-\frac{\partial^{2} \Phi_{j}}{\partial t^{2}} \\
& -\alpha\left(\sum_{i=1}^{n} \frac{\partial \Phi_{j}}{\partial q_{i}} \dot{q}_{i}+\frac{\partial \Phi_{j}}{\partial t}\right)-\beta^{2} \Phi_{j}, \quad j=1, \ldots, r
\end{aligned}
$$

In arriving at representation in Eq. (54), the terms $\alpha$ and $\beta$ are artificially added to improve accuracy and convergence. In the present work, differentiation of the constraints is straightforward but the ensuing equations are quite lengthy and are

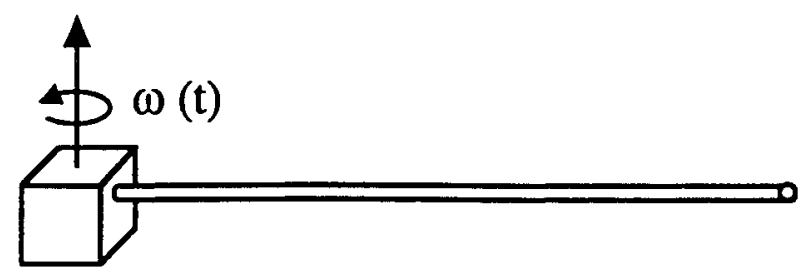

$$
\begin{aligned}
& \rho=250 \mathrm{~kg} / \mathrm{m} \\
& E=6.89 \times 10^{9} \mathrm{~N} / \mathrm{m}^{2} \\
& L=30.5 \mathrm{~m} \\
& A_{b}=9.30 \times 10^{-2} \mathrm{~m}^{2} \\
& I_{3}=7.20 \times 10^{-4} \mathrm{~m}^{4}
\end{aligned}
$$

Fig. 4 Bending validation problem. 
not reproduced here. Interested readers can obtain full details from the first author. For all results presented later, taking $\alpha=\beta=0$ was found to be satisfactory, unless specified otherwise.

\section{Verification Problems, Solutions, and Interpretation}

The failure of the IGC and NSD approaches to solve both bending and membrane dominated problems, and the ability of the AIGC approach to solve both problems will be demonstrated by investigating two verification problems.

A bending-stiffness-dominated problem, studied in Ref. 3, is shown in Fig. 4. It involves a flexible beam cantilevered to a rotating base. The angular velocity of the beam base, which is a prescribed function of time, is given by

$$
\omega(t)= \begin{cases}\omega_{s}\left[6\left(\frac{t}{T_{s}}\right)^{5}-15\left(\frac{t}{T_{s}}\right)^{4}+10\left(\frac{t}{T_{s}}\right)^{3}\right], & \text { if } 0 \leq t \leq T_{s} \\ \omega_{s}, & \text { if } t>T_{s}\end{cases}
$$

where

$$
\begin{gathered}
\omega_{s}=\frac{\pi}{10} \mathrm{rad} / \mathrm{s} \\
T_{s}=1 \mathrm{~s}
\end{gathered}
$$

The lateral deflection response of the free end of the beam given in Ref. 3 is shown in Fig. 5a. The responses for the IGC and NSD approaches (Ref. 8) are given in Fig. 5b, and the response for the AIGC approach is shown in Fig. 5c. Both the IGC and AIGC solutions agree with the results in Ref. 3. However, it is seen that the NSD (even with the inclusion of Yoo's "interaction modes" ${ }^{8}$ ) method fails to correctly solve this problem.

A membrane-stiffness-dominated problem, studied in Ref. 8 is shown in Fig. 6. It is comprised of a flexible beam pinned at the center and outer edge of a rigid rotating table. The prescribed angular velocity of the table is given by

$$
\omega(t)= \begin{cases}\frac{\omega_{s}}{T_{s}}\left[t-\left(\frac{T_{s}}{2 \pi}\right) \sin \left(\frac{2 \pi t}{T_{s}}\right)\right], & \text { if } \quad 0 \leq t \leq T_{s} \\ \omega_{s}, & \text { if } t>T_{s}\end{cases}
$$

where

$$
\begin{gathered}
\omega_{s}=6 \mathrm{rad} / \mathrm{s} \\
T_{s}=15 \mathrm{~s}
\end{gathered}
$$

The solutions predicted by the AIGC, IGC, and NSD approaches, for the lateral deflection of a point at the beam midspan, are shown in Fig. 7. The solutions predicted by the NSD and AIGC approaches are nearly identical and are not individually identifiable. Yoo verified that the solution predicted by the NSD approach, and hence also by the AIGC approach, is correct through the use of an independent calculation of maximum deflection, based on a static structural analogy and an $\mathrm{ADAMS}^{13}$ transient solution. Thus, the solutions shown in Fig. 7 demonstrate the ability of the NSD and AIGC approaches, and the inability of the IGC approach, to solve the membrane-stiffness-dominated problem.

An interesting feature of the results in Fig. 7 is that the steady-state, nonzero lateral deformation predicted by the NSD and AIGC approaches indicates that a buckling-type behavior occurs. Note that this is not predicted by the IGC formulation. The existence of such behavior was verified by a NASTRAN $^{14}$ linear buckling analysis that predicted a first
Lateral Tip Deflection vs. Time

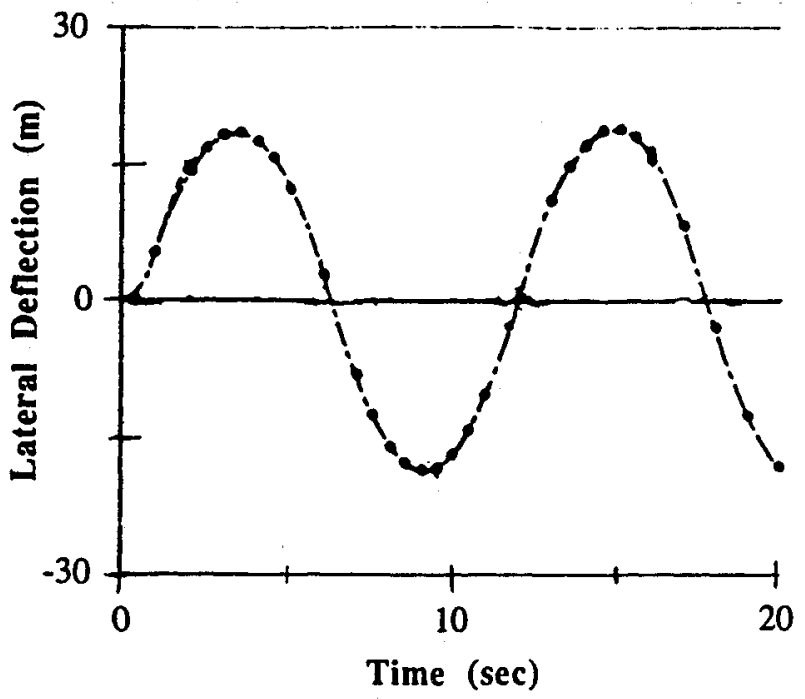

Fig. 5a Christensen's and Lee's results for the bending problem.

\section{Lateral Tip Deflection vs. Time}

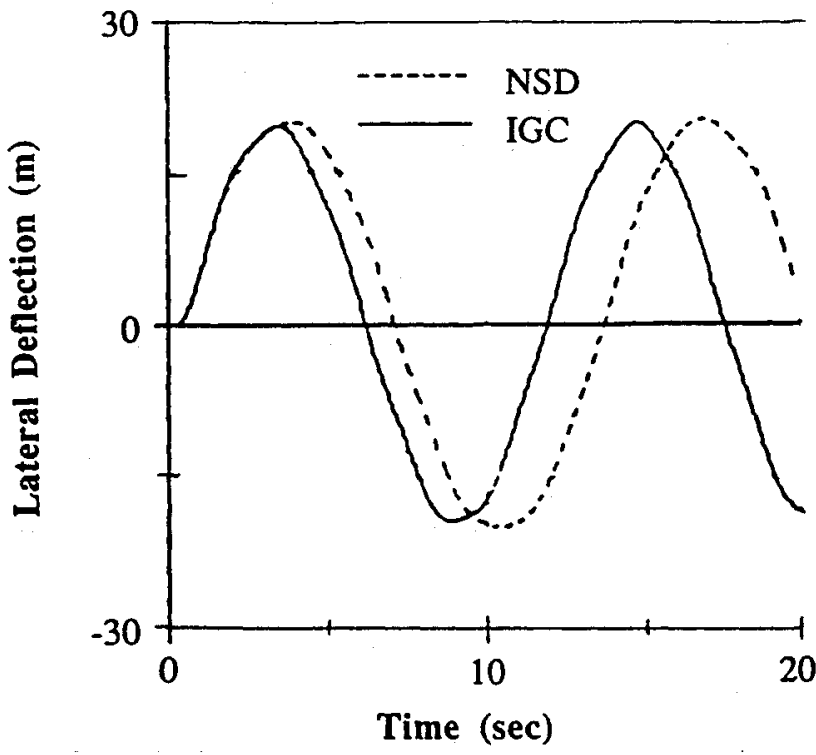

Fig. 5b IGC and NSD results for the bending problem.

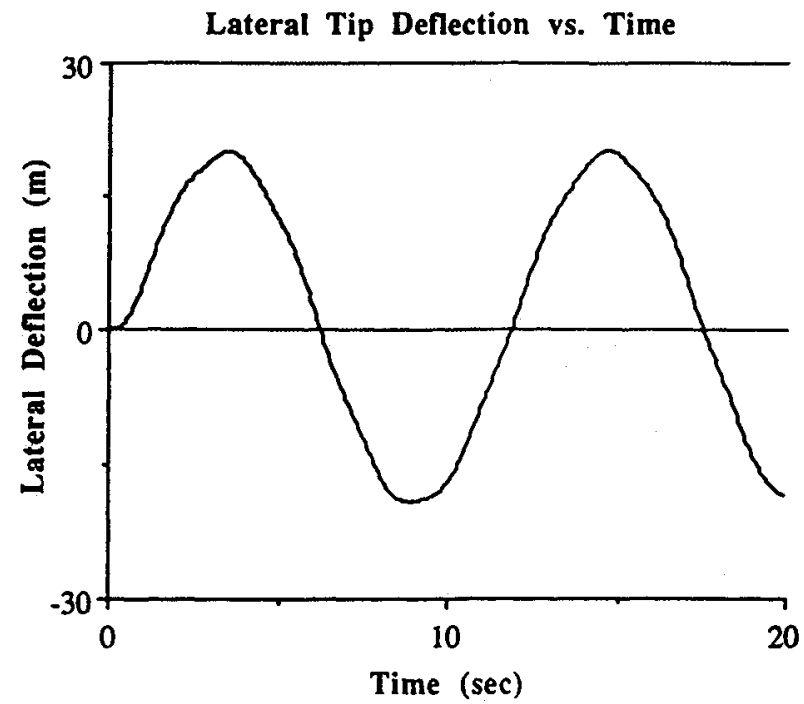

Fig. 5c AIGC results for the bending problem. 


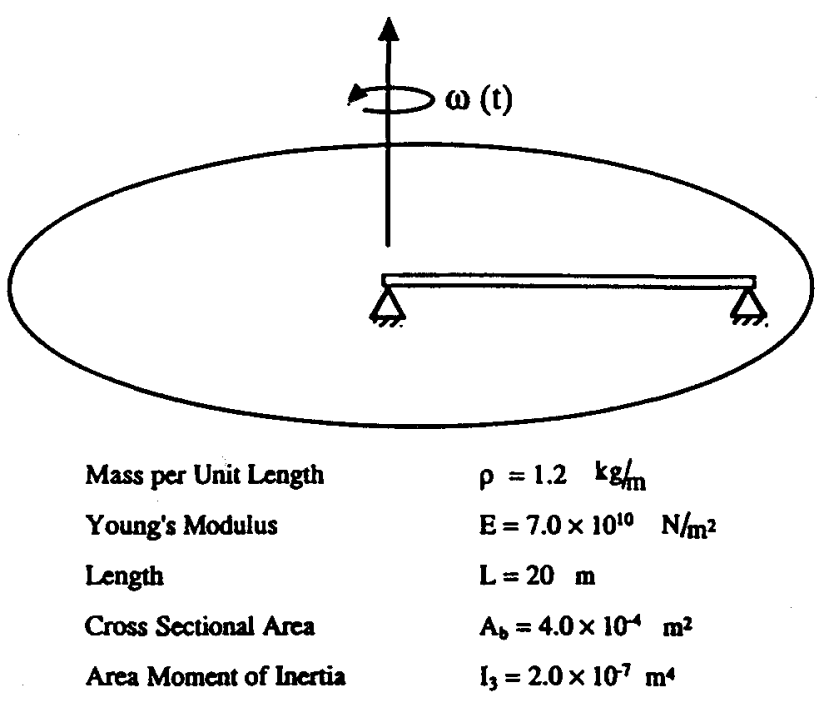

Fig. 6 Membrane validation problem.

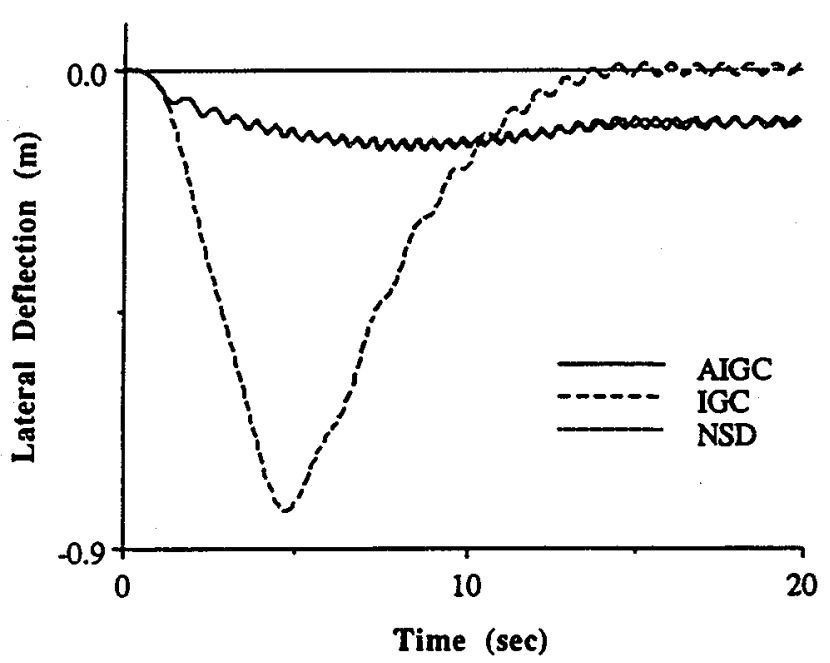

Fig. 7 AIGC, IGC, and NSD results for the membrane problem.

critical angular velocity $\left(\omega_{s}\right)$ of $3.01 \mathrm{rad} / \mathrm{s}$. As seen in Fig. 8 $\left(\omega_{s}=1.0 \mathrm{rad} / \mathrm{s}\right)$, at values of $\omega_{s}$ below the critical speed, no buckling-type behavior is exhibited.

Some other interesting features were also found concerning the membrane-stiffness-dominated problem. Shown in Fig. 9 is the lateral midspan deflection as a function of time for a spin-up time of $3 \mathrm{~s}$, for both undamped (taking $\alpha=\beta=0.2$ was found to be more satisfactory in the damped case) and damped cases (damping was injected by means of a proportional damping assumption; it was added to accentuate the average overall response). Note that the magnitude of the oscillations are larger above the average steady-state value. This clearly shows the nonlinear effect of the membrane stiffness which increases with the absolute value of the lateral deflection.

Finally, it should be noted that more complex behavior can occur. By comparing the AIGC solution shown in Fig. 7 with that shown in Fig. 9, it is seen that the magnitude of the oscillations about the steady-state lateral response increase as the spin-up time decreases. As the spin-up time decreases (below $3 \mathrm{~s}$ ), the value of the midspan deflection (consider the undamped case in Fig. 9) could become positive. The oscillations could then continue about the original buckled position, the beam could perform a continuous snap-through type oscilla-
Lateral Mid-Span Deflection vs. Time

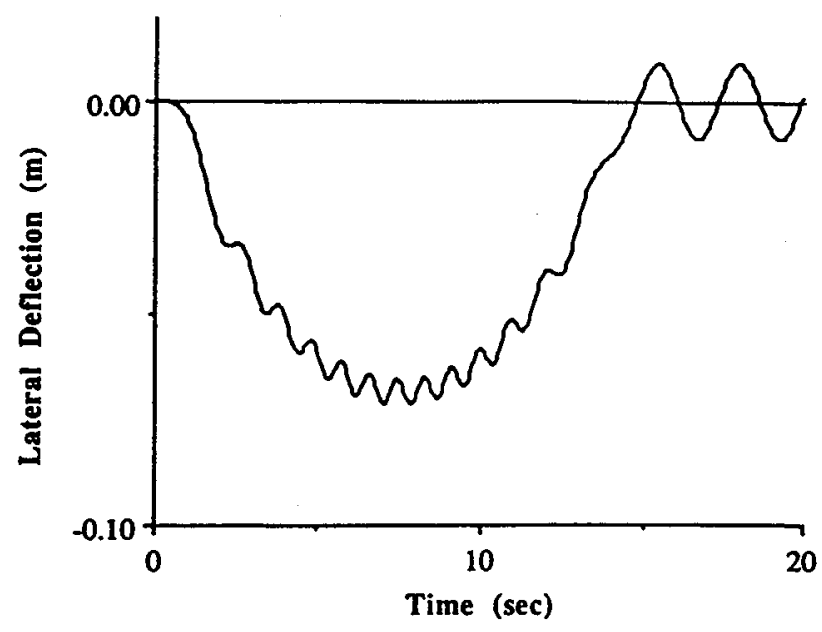

Fig. 8 Membrane problem solution at a subcritical speed.

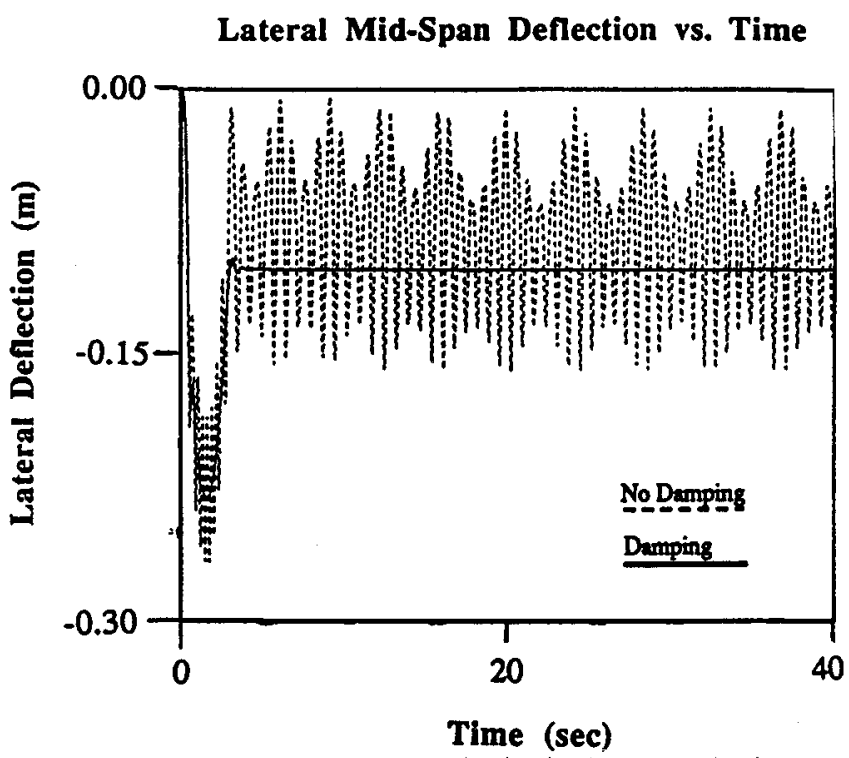

Fig. 9 Membrane problem solution at a spin-up time of $3 \mathrm{~s}$.

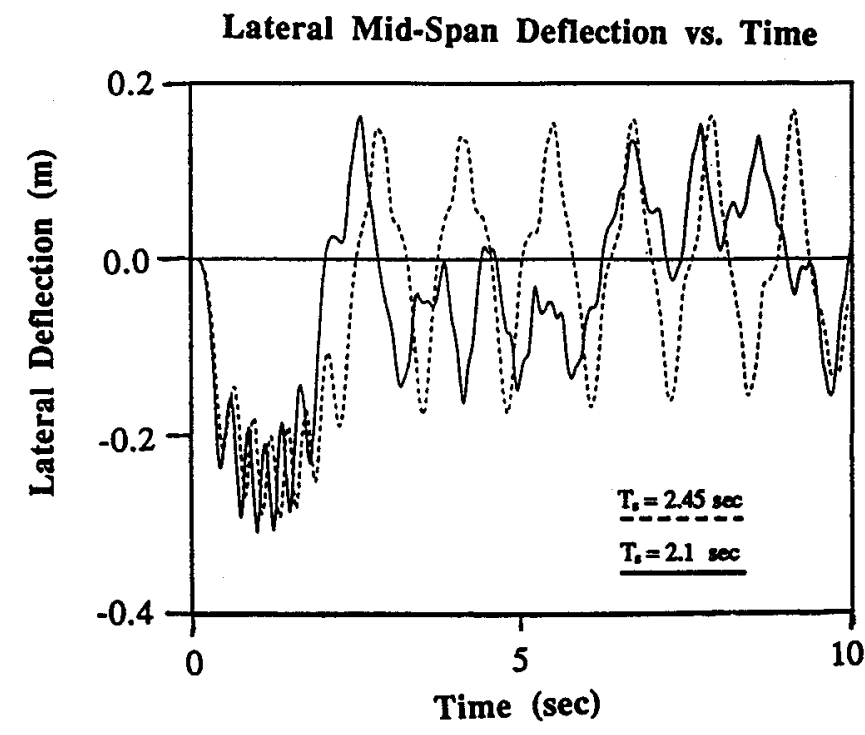

Fig. 10 Two membrane problem solutions for spin-up times less than $3 \mathrm{~s}$. 
tion, or an intermittent type of snap-through oscillation could occur. In Fig. 10, the latter two types of behavior are shown for values of the spin-up time, $T_{s}$, corresponding to $2.45 \mathrm{~s}$ and $2.1 \mathrm{~s}$, respectively.

\section{Extension to Other Physical Problems}

While only problems with cantilevered or pinned-pinned beam attachment have been addressed in this work, problems with other physical attachment could be analyzed with the AIGC approach. This would be done by using the boundary conditions for the specific problem in question. After describing the boundary conditions mathematically in terms of $s, u_{1}$, and $u_{2}$, the appropriate algebraic constraints could be obtained. As is done in this work, the complete equations of motion would then be obtained by addition of the set of second-order differential equations [Eq. (30)]. Although the development of the AIGC approach is limited here to two-dimensional prescribed motion of a single beam, the approach has been extended to three-dimensional motion and deformation as well as applied forces/torques.

\section{Summary}

A new flexible body dynamic beam formulation, the AIGC approach, has been developed. The ability of this approach to improve upon two existing approaches, the IGC and NSD approaches, has been demonstrated through the use of two specific problems. Application of this approach to problems with other boundary conditions has been discussed.

\section{References}

${ }^{1}$ Simo, J. C., "A Finite Strain Beam Formulation. The Three-Dimensional Dynamics Problem. Part I.," Computer Methods in Applied Mechanics and Engineering, Vol. 49, No. 1, 1985, pp. 55-70.
2Simo, J. C., and Vu Quoc, L., "On the Dynamics of Flexible Beams Under Large Overall Motions-The Plane Case: Parts I \& II,' Journal of Applied Mechanics, Vol. 53, No. 4, 1986, pp. 849-863.

${ }^{3}$ Christensen, E. R., and Lee, S. W., "Nonlinear Finite Element Modeling of the Dynamics of Unrestrained Flexible Structures," Computers and Structures, Vol. 23, No. 6, 1986, pp. 819-829.

${ }^{4}$ Kane, T. R., Ryan, R. R., and Banerjee, A. K., "Dynamics of a Cantilever Beam Attached to a Moving Base," Journal of Guidance, Control, and Dynamics, Vol. 10, No. 2, 1987, pp. 139-151.

${ }^{5}$ Banerjee, A. K., and Kane, T. R., "Dynamics of a Plate in Large Overall Motion," Journal of Applied Mechanics, Vol. 56, No. 4, 1989 , pp. 887-892.

${ }^{6}$ Banerjee, A. K., and Dickens, J. M., "Dynamics of an Arbitrary Flexible Body in Large Rotation and Translation," Journal of Guidance, Control, and Dynamics, Vol. 13, No. 2, 1990, pp. 221-227.

${ }^{7}$ Ryan, R. R., "Simulation of Actively-Controlled Flexible Spacecraft," Journal of Guidance, Control, and Dynamics, Vol. 13, No. 4, 1990 , pp. 691-702.

${ }^{8}$ Yoo, H. H., "Dynamic Modeling of Flexible Bodies in Multibody Systems,"' Ph.D. Thesis, Mechanical Engineering, Univ. of Michigan, Ann Arbor, MI, Univ. Microfilms, Inc., 1989.

${ }^{9}$ Kane, T. R., and Levinson, D. A., Dynamics, Theory and Applications, McGraw-Hill, New York, 1983.

${ }^{10}$ Craig, R. R., and Bampton, M. C. C., "Coupling of Substructures for Dynamic Analysis," AIAA Journal, Vol. 6, No. 7, 1968, pp. 1313-1319.

${ }^{11}$ Haering, W. J., "A New Flexible Body Dynamic Formulation for Beam Structures Undergoing Large Overall Motion," Ph.D. Thesis, Mechanical Engineering, Univ. of Michigan, Ann Arbor, MI, Univ. Microfilms, Inc., May 1992.

${ }^{12}$ Baumgarte, J. W., "Stabilization of Constraints and Integrals of Motion in Dynamical Systems," Computer Methods in Applied Mechanics and Engineering, Vol. 1, No. 1, 1972, pp. 1-16.

${ }^{13}$ Anon., ADAMS 5.2 User's Manual, Mechanical Dynamics Inc., Ann Arbor, MI, April 1987.

${ }^{14}$ Anon., MSC/NASTRAN User's Manual Version 66A, The MacNeal-Schwendler Corp., Los Angeles, CA, Nov. 1989. 